\title{
Topology optimization for human proximal femur considering bi-modulus behavior of cortical bones
}

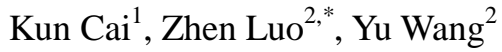 \\ ${ }^{1}$ Science, Information Technology and Engineeering (SITE), University of Ballarat, Mt Helen, Ballarat, VIC 3350 \\ ${ }^{2}$ School of Mechanical and Mechatronic Engineering, The University of Technology, Sydney, NSW 2007 \\ *Author's emails: kuncai99@163.com; zhen.luo@uts.edu.au; Yu.Wang-11@student.uts.edu.au
}

\begin{abstract}
The material in the human proximal femur is considered as bi-modulus material and the density distribution is predicted by topology optimization method. To reduce the computational cost, the bi-modulus material is replaced with two isotropic materials in simulation. The selection of local material modulus is determined by the previous local stress state. Compared with density prediction results by traditional isotropic material in proximal femur, the bi-modulus material layouts are different obviously. The results also demonstrate that the bi-modulus material model is better than isotropic material model in simulation of density prediction in femur bone.
\end{abstract}

Keywords: Topology optimization, proximal femur, bone remodelling, multiple load cases, bi-modulus material

\section{Introduction}

Osteoporosis, a kind of bone illness, happens widely in aged people. Serious osteoporosis leads to fracture of bone happening easily. To give an efficient therapy of osteoporosis (e.g., improving the speed of bone apposition), the mechanical properties of (both of cortical and cancellous) bone should be investigated and some results may act an important role in therapy of osteoporosis. In research of bone mechanics, the Wolff's law (Wolff 1892), which states that bone micro-structure (Figure 1) and local stiffness tend to align with the principal stress directions according to its mechanical environments, is actually a core concept. In the viewpoints of some researchers, the bone is an optimal structure with "maximum" mechanical efficiency but "minimum" mass according to the Wolff's law. During the last 40 years experimental equipments and computational techniques have been developed rapidly. Nowadays, the quantitative study of the Wolff's law can be carried out both of experimentally and numerically.

During the last four decades, the Wolff's law has been validated by many investigations. Much attention has been paid on the relations between the loading environments and the micro-structure of bone (Whitehouse and Dyson 1974; Cowin 1986; Odgarrd et al. 1997; Zhu et al. 2006; Cory et al. 2010; Nazarian et al. 2011).

It is interesting that bone modelling process is often considered as an optimization process. Hollister et al. (1993) tried to answer the question: whether bone remodelling can approach a globally optimized structure. Confined by computational conditions, the conclusion was not positive. Later, Jang and Kim (2008) quantitatively investigated the validity of Wolff's law by topology optimization method. They claimed that topology optimization (with minimal structural compliance) and the bone remodeling (SED distributing uniformly) are equivalent. Fernandes et al. (1999) an analytical parametric micro-structural model for trabecular bone in proximal femur was proposed according to the homogenization theory, and optimal densities and orientations were obtained by topology optimization. Similar work was given by Kowalczyk (2010). Design space optimization (DSO) method by (Kim and Kwak 2002) was adopted for micro-structure prediction of proximal femur by their group (Kim et al. 2008; Jang and Kim 2008).

Zhu et al. (2006) tested the tensile and compressive modulus of Takin femoral cortical bone. They found that the compressive modulus of bone is 5-6 times of the tensile modulus. The difference also exists in other bones (Cory et al., 2010; Nazarian et al., 2011). However, till now, no work gives the 
prediction of cancellous bone in proximal femur with consideration of the bi-modulus behavior of bone. In this study, a material replacement method (Cai et al. 2013) is used to investigate the optimal material layout in proximal femur.

\section{Material Properties}

\subsection{Bi-modulus behavior of elastic material}

Experiments (Zhu et al, 2006) show a cortical bone has different elastic behaviors under tension and compression load. Therefore, bone is a typical bi-modulus material. Figure 2 gives the stress-strain curve of a bi-modulus material. Tangent $\alpha$ gives the tension modulus of material, i.e., $E_{T}=\tan \alpha$; and the tangent $\beta$ is the compression modulus, i.e., $E_{C}=\tan \beta$. The ratio between them are marked with $R$, e.g., $R=E_{T} / E_{C}$. The material becomes an isotropic material when $\alpha=\beta$. Clearly, bi-modulus material properties are stress-dependent. Usually, in structural analysis, the piece-wise linear material has to be treated as nonlinear and approximated with differentiable curve in structural reanalysis (Medri 1982). But in the present work, the character of the curve, i.e., piecewise linear, is adopted by material replacement operation (Cai et al., 2013), i.e., the bi-modulus material is replaced with two isotropic material and one of them will be used for an element in finite element analysis according to the previous stress state of the element.

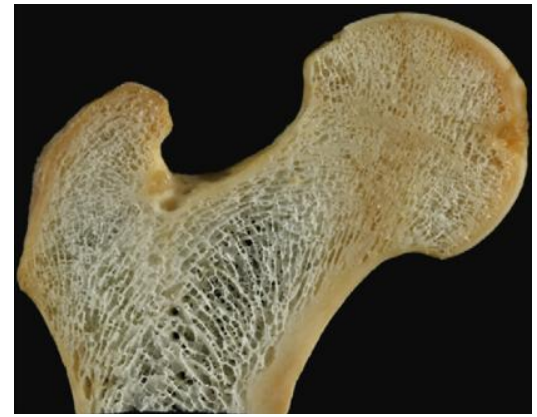

Figure 1. Photograph and radiograph of human proximal femur (Skedros and Baucom 2007)

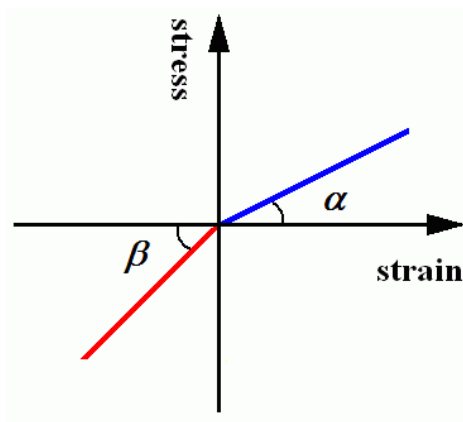

Figure 2. The stress-strain curve of a bi-modulus material, $\alpha \neq \beta$

\subsection{Stiffness of porous material}

For porous material (e.g., the $m$-th material sample or finite element), the relationship between the stiffness tensor and the volume fraction (relative density) is expressed as

$$
D_{m, i j k l}=\rho_{m}^{p} D_{0, i j k l}
$$

Where $D_{m, i j k l}$ is the elastic tensor of porous material, and the relative density $\rho_{m} \in\left[\begin{array}{ll}0 & 1.0\end{array}\right]$. According to experiments data of bone (Buchler et al. 2002), the power coefficient $p$ is set to be 2 in the present study. $D_{0, i j k l}$ is the elastic tensor of fully solid material.

\section{Optimization model}

\subsection{Formulations of topology optimization problem}

Continuum topology optimization method became a hot point in structural optimization since the homogenization design method (HDM) was presented by (Bendsøe and Kikuchi 1988). A large number of efforts have been paid on the development of continuum topology optimization methods over the past two decades. Besides the HDM, the most popular methods are, the solid isotropic micro-structures with penalization (SIMP) method (Rozvany et al. 1992; Bendsøe and Sigmund 1999); the nodal density description method (Matsui and Terada 2004; Kang and Wang 2012; Luo et al. 2013); the evolutionary structural optimization (ESO) method by (Xie and Steven 1993); and the level set method by (Wang et al. 2003; Allaire et al. 2004; Luo et al. 2007).

In the present work, the final density distribution of bone in proximal femur is obtained by modified 
SIMP method, rather than bone remodeling model. The volume constrained optimization of a structure with minimum of the structural mean compliance under multiple load cases, is expressed as

$$
\begin{aligned}
& \min _{\left\{\rho_{m}\right\}} c=\sum_{i}^{N} w_{i} \boldsymbol{U}_{i}^{T} \overline{\boldsymbol{K}_{i}} \boldsymbol{U}_{i}=\sum_{i}^{N} w_{i} \sum_{m=1}^{M}\left(\boldsymbol{u}_{m}^{T} \overline{\boldsymbol{k}}_{m} \boldsymbol{u}_{m}\right)_{i} \\
& \text { s.t. } \sum_{i}^{N} \sum_{m=1}^{M}\left(\rho_{m} v_{m}\right)_{i}=f_{v} \cdot V_{0} \\
& \boldsymbol{K}_{i} \boldsymbol{U}_{i}=\boldsymbol{P}_{i}, i=1,2, \cdots, N \\
& 0<\rho_{\text {min }} \leq \rho_{m} \leq 1.0, m \in \Omega
\end{aligned}
$$

where the objective function $c$ is the sum of the structural mean compliance. $N$ is the number of load cases the structure subjected to. $w_{i}$ is the weighted coefficient for the $i$-th load case. $M$ is the total number of elements. $\left\{\rho_{m}\right\}$ is the set of relative densities of elements. $\boldsymbol{U}_{i}$ and $\boldsymbol{P}_{i}$ are the global nodal displacement and force vectors in the $i$-th load case, respectively. $\overline{\boldsymbol{k}}_{m}$ is the modified matrix of $\boldsymbol{k}_{m}$ (the stiffness matrix of the $m$-th element with isotropic material). The global stiffness matrix of structure $\boldsymbol{K}_{i}$ is assembled with $\left\{\boldsymbol{k}_{m}\right\}_{i} \cdot \overline{\boldsymbol{K}}_{i}$ is assembled with $\left\{\overline{\boldsymbol{k}}_{m}\right\}_{i} \cdot \boldsymbol{u}_{m}$ is the nodal displacement vector of the $m$-th element. $v_{m}$ is the volume of the $m$-th element. $f_{v}$ is the critical volume ratio of the final structure. $V_{0}$ is the total volume of solid design domain.

\subsection{Selection criterion of elastic modulus of material}

After being replaced with two isotropic materials, the original bi-modulus material in an element will be considered as one of the two isotropic materials for structural analysis. The elastic modulus of an element in structure under MLC is determined by the following equation

$$
E_{m}=\left\{\begin{array}{l}
E_{T}, \quad \text { if } \quad(\mathrm{TSED}>\mathrm{CSED})_{m} \\
E_{C}, \quad \text { if }(\mathrm{TSED}<\mathrm{CSED})_{m} \\
\max \left(E_{T}, E_{C}\right), \quad \text { others }
\end{array}\right.
$$

where the TSED (tension strain energy density) and CSED (compression strain energy density) can be calculated by the following formulations

$$
\begin{aligned}
\operatorname{TSED}_{m} & =\frac{1}{4} \sum_{i=1}^{N} \sum_{j=1}^{3}\left(\sigma_{j, i}+\left|\sigma_{j, i}\right|\right) \cdot \varepsilon_{j, i} \\
\operatorname{CSED}_{m} & =\frac{1}{4} \sum_{i=1}^{N} \sum_{j=1}^{3}\left(\sigma_{j, i}-\left|\sigma_{j, i}\right|\right) \cdot \varepsilon_{j, i}
\end{aligned}
$$

Where $\sigma_{j, i}$ and $\varepsilon_{j, i}$ are the mean principal stress and strain of element, respectively.

\section{Result and Discussions}

The deformation analysis in topology optimization of proximal femur is accomplished with the commercial software ANSYS (2013) and 4-node plane stress element is used.

\subsection{Finite element model for proximal femur}

Table 1. Forces in three load cases on the proximal femur model. Orientations are given according to vertical (negative for left and positive for right). Negative force means compression and positive means tension (Jang and Kim 2008)

\begin{tabular}{|c|c|c|c|c|c|}
\hline \multirow{2}{*}{ Load case } & \multirow{2}{*}{ Cycles/day } & \multicolumn{2}{|c|}{ Abductor reaction } & \multicolumn{2}{c|}{ Joint reaction } \\
\cline { 3 - 6 } & & Magnitude/N & Orientation $/{ }^{\circ}$ & Magnitude/N & Orientation ${ }^{\circ}$ \\
\hline 1(one-legged & 6000 & 703 & 28 & -2317 & 24 \\
\hline
\end{tabular}




\begin{tabular}{|c|c|c|c|c|c|}
\hline stance) & & & & & \\
\hline 2 (abduction) & 2000 & 351 & -8 & -1158 & -15 \\
\hline 3(adduction) & 2000 & 468 & 35 & -1548 & 56 \\
\hline
\end{tabular}

The upper part of proximal femur of human (see Figure 3a) is discretised into 9240 elements. The bottom is simply supported. In this study, the cortical bone with tensile elastic modulus of $17.0 \mathrm{GPa}$ (the mean value) and Poisson's ratio of 0.3 (Gupta et al., 2004.). The relative density of bone varies within the interval $[0.051 .0]$. The modulus of bone under tension is not greater than that under compression. And the ratios between tension and compression moduli are as follows. $R=1.0$ and 0.5 , respectively. As $R$ equals 1.0, the material shows isotropic. At the same time, two cases on considering the volume ratio of proximal femur are discussed, e.g., $35 \%$ and $46 \%$. From the data in Table 1 , the three weighted coefficients are $0.6,0.2$ and 0.2 for three cases, respectively.

\subsection{Optimal material distributions in proximal femur}

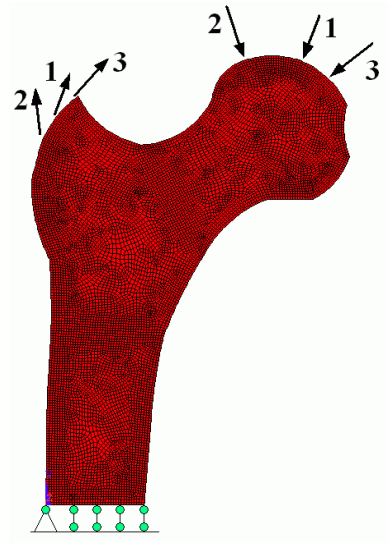

(a) three load cases

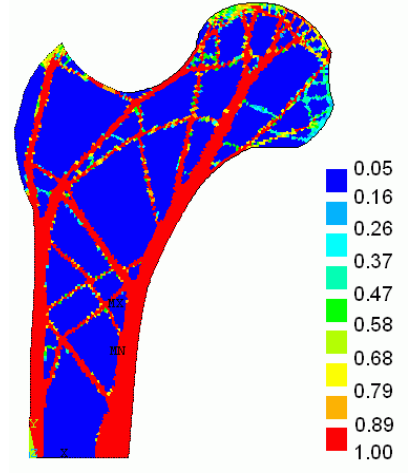

(b) density plot as $f_{v}=35 \%$

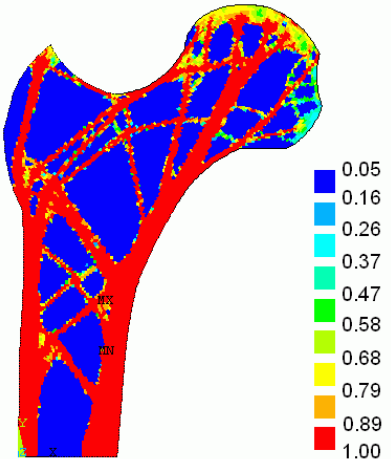

(c) density plot as $f_{v}=46 \%$

Figure 3. Finite element of proximal femur (a) and density distributions of isotropic material $(R=1)$

Figure $3 \mathrm{~b}$ and $\mathrm{c}$ give the final isotropic material distributions in proximal femur with different volume ratios. The amount of material supporting the first load case (one-legged stance) increase obviously. It reflects the real loading activity one proximal femur.

Figure 4 gives the final bi-modulus material $(R=0.5)$ distributions in proximal femur with different amount of residual material. The amounts of material with mid-density are greater than those in Figure $3 b$ and $c$, respectively. Comparing with Figure $3 b$ and $c$, the result in Figure $4 b$ gives a better prediction of cancellous distribution in femur bone (Figure 1).

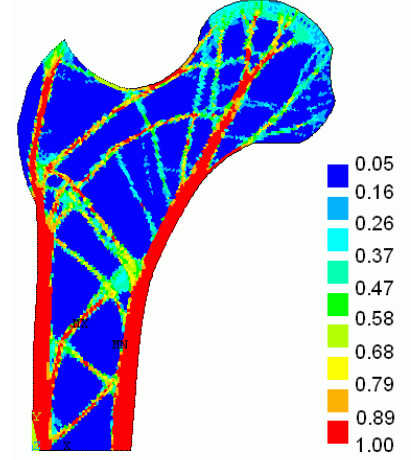

(b) density plot as $f_{v}=35 \%$

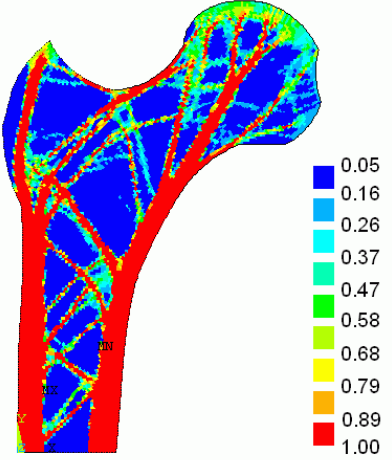

(b) density plot as $f_{v}=46 \%$

Figure 4. density distributions of isotropic material $(\boldsymbol{R}=0.5)$ in proximal femur

\section{Conclusions}


In simulation of density distribution of the human proximal femur, the material constitutive model is essential to the results. From the results given above, the bi-modulus material model matches the real architecture better than isotropic material model. As the finer finite element mesh is adopted, a perfect detailed architecture of trabecular in femur bone can be found.

\section{Acknowledgements}

The financial supports of the National Natural Science Foundation of China (Grant No. 50908190, 51179164) and the Fundamental Research Foundation of Northwest A\&F University (Grant No. QN2011125) are greatly acknowledged.

\section{References}

Ansys, Inc., Ansys, 2013. http://www.ansys.com.

Bendsøe, M.P., Kikuchi, N., 1988. Generating optimal topologies in structural design using a homogenization method. Computer Methods in Applied Mechanics and Engineering, 71, 197-224.

Bendsøe, M.P., Sigmund, O., 1999. Material interpolation schemes in topology optimization. Archive of Applied Mechanics, 69, 635-654.

Buchler, P., Ramaniraka, N.A., Rakotomanana, L.R., Iannotti, J.P., Farron, A., 2002. A finite element model of the shoulder: application to the comparison of normal and osteoarthritic joints. Clinical Biomechanics 17(9-10), 630-639.

Cai, K., Gao, Z.L., Shi, J., 2013. Compliance optimization of a continuum with bi-modulus material under multiple load cases. Computer-Aided Design 45, 195-203.

Cory, E., Nazarian, A., Entezari, V., Vartanians, V., Muller, R., Snyder, B.D., 2010. Compressive axial mechanical properties of rat bone as functions of bone volume fraction, apparent density and micro-ct based mineral density. Journal of Biomechanics 43, 953-960.

Cowin, S.C., 1986. Wolff's law of trabecular architecture at remodeling equilibrium. Journal of Biomechanical Engineering 108, 83-88.

Fernandes, P., Rodrigues, H., Jacobs, C., 1999. A model of bone adaptation using a global optimization criterion based on the trajectorial theory of Wolff. Computer Methods in Biomechanics and Biomedical Engineering 2(2), 125-138

Gupta, S., vander Helm, F.C., van Keulen, F., 2004. The possibilities of uncemented glenoid component-a finite element study. Clinical Biomechanics 19(3), 292-302.

Hollister, S.J., Kikuchi, N., Goldstein, S.A., 1993. Do bone ingrowth processes produce a globally optimized structure? Journal of Biomechanics 26(4-5), 391-407.

Luo, Z., Tong, L., Wang, M.Y., Wang, S., 2007. Shape and topology optimization of compliant mechanisms using a parameterization level set method. Journal of Computational Physics, 227, 680-705

Luo, Z., Zhang, N., Wang, Y., Gao, W., 2013. Topology optimization of structures using meshless density variable approximants. International Journal for Numerical Methods in Engineering, 93, 443-464.

Jang, I.G., Kim I.Y., 2008. Computational study of Wolff's law with trabecular architecture in the human proximal femur using topology optimization. Journal of Biomechanics 41, 2353-2361.

Kang, Z., Wang, Y.Q., 2011. Structural topology optimization based on non-local Shepard interpolation of density field. Computer Methods in Applied Mechanics and Engineering, 200, 3515-3525.

Kim, H.A., Clement, P.J., Cunningham, J.L., 2008. Investigation of cancellous bone architecture using structural optimisation. Journal of Biomechanics 41, 629-635.

Kim, I.Y., Kwak, B.M., 2002. Design space optimization using a numerical design continuation method. International Journal for Numerical Methods in Engineering 53, 1979-2002.

Kowalczyk, P., 2010. Simulation of orthotropic micro-structure remodelling of cancellous bone. Journal of Biomechanics 43, 563-569.

Matsui, K., Terada, K., 2004. Continuous approximation of material distribution for topology optimization. International Journal for Numerical Methods in Engineering, 59 1925-1944.

Medri, G., 1982. A nonlinear elastic model for isotropic materials with different behavior in tension and compression. Transactions of the ASME 104(1), 26-28.

Nazarian, A., Araiza Arroyo, FJ., Rosso, C., Aran, S., Snyder, B.D., 2011. Tensile properties of rat femoral bone as functions of bone volume fraction, apparent density and volumetric bone 
mineral density. Journal of Biomechanics 44, 2482-2488.

Odgaard A., Kabel, J., van Rietbergen, B., Dalstra, M., Huiskes, R., 1997. Fabric and elastic principal directions of cancellous bone are closely related. Journal of Biomechanics, 30, 487-495.

Rozvany, G.I.N., Zhou, M., Birker, T., 1992. Generalized shape optimization without homogenizaiton. Structural Optimization 4, 250-252.

Skedros, J.G., Baucom, S.L., 2007. Mathematical analysis of trabecular 'trajectories' in apparent trajectorial structures: the unfortunate historical emphasis on the human proximal femur. Journal of Theoretical Biology 244, 15-45.

Wang, M.Y., Wang, X., Guo, D., 2003. A level set method for structural topology optimization. Computer Methods in Applied Mechanics and Engineering 192, 227-46.

Whitehouse, W.J., Dyson, E.D., 1974. Scanning electron microscope studies of trabecular bone in the proximal end of the human femur. Journal of Anatomy 118 (3), 417-444.

Wolff, J., 1892. Das Gesetz Der Transformation Der Knochen. Hirschwald, Berlin.

Xie, Y.M., Steven, G.P., 1993. A simple evolutionary procedure for structural optimization. Computers and Structures 49, 885-896.

Zhu, L., Zhao, H.P., Song, Y.L., Feng, X.Q., 2006. Experimental investigation of the mechanical properties of Takin femoral cortical bone. Journal of Tsinghua University(Sci \& Tech) 46(2), 301-304. 